CITATION: Patellofemoral pain: Challenging current practice - A case report. Smith, Benjamin E. et al.Manual Therapy , Volume 22 , 216- 219 DOI: http://dx.doi.org/10.1016/i.math.2015.09.002

\title{
Patellofemoral pain: Challenging current practice - A case report
}

Benjamin E. Smith, Derby Teaching Hospitals NHS Foundation Trus, Paul Hendrick, Pip Logan, University of Nottingham

\section{ABSTRACT}

Patellofemoral pain (PFP) is a common problem in young people, with 1 in 6 suffering at any one time. It is unclear which management approach is the optimal method for treating PFP in the long term, with traditional physiotherapy examination focusing on assessing for specific structural dysfunction. A rationale for a different assessment and treatment approach, one that moves the focus away from a biomedical/tissue pathology model towards one directed at the neurophysiology of pain, has been suggested.

The patient was a 21 year old male with a 6 year history of PFP with previous failed physiotherapeutic treatment. He reported previous multiple healthcare practitioners' advice to avoid activities that were painful as reasons for being unable to participate in sporting activities. No specific structural testing was performed, such as specific muscle strength, length, foot position, patella movement and position, or movement patterns.

Descriptions of tissue based pathology models of pain, e.g. patella mal-tracking, were actively discouraged and challenged. The patient was taught to perform one uncomfortable/painful exercise as part of his rehabilitation programme twice a day.

The patient achieved $80 \%$ improvement in his symptoms over 7 appointments and a return to physical activity following a 5 month rehabilitation programme purposively designed to elicit pain by means of gradually exercising and loading the tissues. This case report highlights the need for further research into exercise protocols for patients suffering with PFP based upon neurophysiology models of pain. 